EFI-02-85

hep-th/0206175

\title{
Exciting AdS Orbifolds
}

\author{
Emil J. Martined and Will McElgin[ \\ Enrico Fermi Inst. and Dept. of Physics \\ University of Chicago \\ 5640 S. Ellis Ave., Chicago, IL 60637, USA
}

\begin{abstract}
The supersymmetric $\left(A d S_{3} \times S^{3}\right) / \mathbb{Z}_{N}$ orbifold constructed by the authors in hep-th/0106171 is shown to describe AdS fragmentation, where fivebranes are emerging from the F1-NS5 background. The twisted sector moduli of the orbifold are the collective coordinates of groups of $n_{5} / N$ fivebranes. We discuss the relation between the descriptions of this background as a perturbative string orbifold and as a BPS state in the dual spacetime CFT. Finally, we attempt to apply the lessons learned to the description of BTZ black holes as $A d S_{3}$ orbifolds and to related big crunch/big bang cosmological scenarios.
\end{abstract}

\footnotetext{
${ }^{1}$ e-martinec@uchicago.edu

2 wmcelgin@theory.uchicago.edu
} 


\section{Introduction and Summary}

Much interesting structure of string theory has been revealed through the investigation of its asymptotically $A d S$ backgrounds - the precise counting of black hole microstates [1]; the matching of low-energy black hole emission/absorption spectra between string theory and supergravity [2, 3]; the UV/IR connection [4]; and spacetime topology changing transitions [5]-[7], to name but a few. The overarching idea encompassing all of these results is the duality between quantum gravity and a holographically dual conformal quantum field theory [8].

The $A d S_{3}$ example has figured prominently in these discussions; it arises as the near-horizon geometry of $n_{1}$ strings and $n_{5}$ fivebranes. In addition to the above list of results, it has also proved a fruitful ground for exploring additional issues, such as the black hole information paradox (see [?]-[12] for a few examples), and the role of singular conformal field theories [13].

One distinctive feature of $A d S_{3}$ is that the supergravity side of the correspondence admits a perturbative string description at points in moduli space with vanishing RR fields [14], giving one access to more than just the low-energy supergravity spectrum and dynamics on the 'bulk' side of the correspondence. Another is that the current algebraic nature of the conformal symmetry provides more powerful tools in the analysis of the 'boundary' theory - in this case a sigma model on the moduli space of instantons [15, 16, 17].

Given the degree of control one has over both sides of the correspondence for $A d S_{3} / C F T_{2}$, one may ask whether it is possible to study black hole physics while having simultaneous control over both a bulk and boundary description of the dynamics, for instance to see the 'long string modes' describing black hole states [18] in a description that also has control over local physics in the bulk, such as that of [14]. It was with this goal in mind that the authors began a study of $A d S_{3} \times S^{3}$ orbifolds in [19], in part because of the naive interpretation of the $A d S_{3}$ or BTZ black hole [20, 21] as an orbifold of global $A d S_{3}$ spacetime.

The orbifold procedure is a useful way of generating new backgrounds from solvable examples. Where the orbifold operation takes the theory seems to be strongly example-dependent. For example, a chiral $\mathbb{Z}_{N}$ orbifold of the $S^{3}$ has the interpretation of adding KK monopole charge to the background [22]. The effect on the dual CFT of this orbifold operation is not known (see 23 for a discussion).

In [19], it was shown that the bulk orbifold $\left(A d S_{3} \times S^{3}\right) / \mathbb{Z}_{N}$ (by opposite $\mathbb{Z}_{N}$ rotations in both $S^{3}$ and in the spatial directions of $A d S_{3}$ ) yields a BPS state in the same spacetime CFT that describes the original global $A d S_{3}$ spacetime as its $S L(2)$ invariant vacuum state. Reasons for considering this orbifold include (i) the technology for time-independent orbifolds is well-understood; and (ii) naively the geometry approaches the extremal BTZ black hole threshold from below as $N \rightarrow \infty$, so one might hope to have a situation where there is some degree of control over both geometry and black hole degrees of freedom.

In this work, we examine the latter construction in more detail, in particular 
we find in section 2 precisely which among a large degeneracy of BPS states is being described by the bulk orbifold. It will turn out that the configuration being described is that of $N$ groups of $n_{5} / N$ fivebranes near the point where they emerge onto the Coulomb branch of their moduli space ${ }^{5}$. We further give an interpretation of the various excitations of the orbifold in section 3 - especially the twisted sectors, where the twisted sector moduli are shown to describe the relative separations of the fivebranes.

It also turns out that there is a remarkable parallel between the description of the rotational orbifold in the bulk perturbative string theory and the description of the twist vertex operators in the dual boundary CFT that create the corresponding BPS states from the $S L(2)$ invariant vacuum. Namely, the twist operator correlation functions have the same geometrical description in terms of the vacuum-to-vacuum amplitude on a branched cover of the Riemann sphere (the Euclidean $A d S_{3}$ boundary), as the bulk orbifold does in terms of its branched cover onto the global $A d S_{3}$ spacetime!

After a brief comment on related fivebrane backgrounds in section 1 , we turn in section 5 to a discussion of the $A d S_{3}$ black hole geometry as an orbifold [20, 21]. After reviewing the geometry of the identification, we discuss the nature of the vacuum and in particular its behavior near the black hole singularities.

As a classical geometry, the eternal $A d S_{3}$ (BTZ) black hole is a quotient of the $A d S_{3}$ geometry by an element of the $S L(2, \mathbb{R}) \times S L(2, \mathbb{R})$ isometry group that roughly speaking acts as a boost rather than a rotation. Thus, a freely falling observer experiences a geometry that near the singularity looks like a sort of Milne universe. Such geometries have been periodically identified as interesting subjects for investigation, both in the context of perturbative string theory and in quantum cosmology [25]- 40]. The group action identifies the boundary of $A d S_{3}$ in such a way that the intersection of the black/white hole singularities with the boundary are again locally Milne singularities, now in the $1+1$ dimensional CFT on the Lorentzian cylinder which is the conformal boundary of $A d S_{3}$. If one considers $S L(2, \mathbb{R})$ rather than its covering space, there are four such singularities (two if one further identifies to the Poincaré patch $P S L(2, \mathbb{R}))$.

The advantage of an $A d S_{3}$ based example is again that one may be able to bring to bear both a perturbative bulk (string) description of the BTZ orbifold [25, 26, 27, 35, 36, 37, 39] as well as a two-dimensional boundary CFT description. As discussed above, in matching the two dual descriptions, one must overcome the obstacle that it is not always known how the orbifold procedure affects the boundary theory. This is especially true when we abandon orbifold actions that preserve sufficient supersymmetry to guarantee some amount of nonrenormalization of the background, since we lose control over what properties of tree-level string theory are

\footnotetext{
${ }^{3}$ The Coulomb branch for fivebranes is lifted at generic points of the $O(5,4) / O(5) \times O(4)$ moduli space of supergravity in the F1-NS5 background, but it is present at the locus of vanishing RR fields described by the perturbative string formalism of [14]. This locus of moduli space characterizes the singular CFT's of [24, 13, 23, 14].
} 
maintained in the full nonperturbative quantum state; there is no guarantee that string perturbation theory is an accurate guide to the exact geometry. Quantum corrections could completely invalidate the picture of the geometry as an orbifold, i.e. as something that can be covered onto the global $A d S_{3}$ geometry. Nevertheless, we proceed under the assumption that states in the boundary theory which respect the appropriate discrete symmetries will possess the same basic characteristics as the Kruskal vacuum of the BTZ geometry, and attempt to deduce some of their properties. If this assumption proves incorrect, then one cannot characterize the BTZ black hole as an $A d S_{3}$ orbifold.

A persistent question in the AdS/CFT correspondence has been how to describe propagation into the black hole singularity within the framework of the dual CFT; a related source of confusion is what it means to propagate through the horizon of the extremal brane geometry according to the global time rather than the Poincaré time naturally related to the brane Hamiltonian (see for example [10]). A fundamental issue is how to treat the intersection of the horizon (and/or the singularity) with the conformal boundary. In fact, it is generally true that the outer horizon, inner horizon, and singularity of any BTZ black hole all coincide at the boundary of the $A d S$ covering space. The transformation between the global and Poincaré coordinates, or between global and BTZ Kruskal coordinates, has singularities at these points; blithely adding the singular locus to the conformal compactification of the Poincaré patch or the Kruskal boundary is not always justified. In the present context, the question becomes whether the states in the boundary theory that respect the (extremal or nonextremal) BTZ identification have a sensible description in the Hilbert space of eigenstates of the global time, so that one can make sense of propagating them through the Milne singularities. A reasonable prescription for dealing with such singularities in $1+1$ dimensional CFT would go a long way toward justifying recent speculations about cosmological singularities by providing them with an interpretation in a fully quantum mechanical realization of gravity, via the interpretation of the CFT as the boundary dual to asymptotically AdS quantum gravity.

We will find that the stress tensor of the required CFT state diverges rather badly along the light cones emanating from the singular locus on the boundary, presenting a difficulty for the orbifold characterization of the black hole, and associated cosmological models. Such a divergence might be regarded as an instance of the generic divergence of stress tensors on Cauchy horizons (c.f. 41] and references therein).

We conclude this introduction with a comment on the apparent diversity of presentations of the BTZ black hole. There are by now many descriptions of CFT states purportedly dual to the black hole - the thermal state in the Hilbert space

\footnotetext{
${ }^{4}$ The same assumption is implicit in the work of [28], where even more exotic identifications of $A d S_{3}$ are considered in the context of cosmology. The discrete subgroups of $S L(2, \mathbb{R})$ considered there are allowed to act discontinuously on the conformal boundary - the parameter space of the boundary CFT is a noncommutative geometry (G. Horowitz and M. Douglas, unpublished).
} 
$\mathcal{H}$ of the CFT; the entangled state in $\mathcal{H}^{\otimes 2}$ suggested by the Kruskal geometry; states formed by collapse of energetic states in supergravity; and the hyperbolic orbifold. Are they all the same black hole geometry? Our view on this issue is that they are not. They are distinguished by the history along the entire spacetime boundary which they possess. For instance, a generic state in the thermal ensemble, or one formed by the collapse of energetic probes dropped from the AdS boundary, employ a single CFT Hilbert space $\mathcal{H}$ and describe a spacetime with one asymptotic boundary; while the correlated state in $\mathcal{H}^{\otimes 2}$ of [12 describes a spacetime with two disjoint asymptopia. Any set of observations made on a single asymptotic boundary in any of these examples will find what semiclassically looks like a black hole. There is a certain artificiality to the AdS/CFT correspondence when viewed cosmologically, in that there is an external agent who sets up the 'universe' under consideration. The generic high energy eigenstate state in $\mathcal{H}$ will be approximately thermal in its properties, and does not arise from a collapse process, but still looks like a black hole. The universe one 'creates' may have one asymptotic boundary, as in the case of the thermal CFT state; or it may have two, as in the case of the entangled state of the Kruskal extension (it may also have many, or possibly none [28]!). It may not be possible to detect the full geometry on the basis of experiments available to any single observer, but that does not invalidate the cosmology until and unless one can develop criteria to eliminate these more exotic examples with multiple holographic screens. This observation may also have consequences for de Sitter cosmology, where the supposition of a holographic dual containing only a horizon area's worth of states assumes that there is no part of the world holographically inaccessible to a given observer. Finally, the ability to cover cosmologies with closed spatial geometry onto global AdS spacetime may indicate the presence of non-obvious and/or nonlocal observables (c.f. [40]), whose apparent lack has long been a stumbling block in understanding closed quantum cosmologies. The answer to these questions bear upon whether intial conditions with cosmological past horizons are admissible, and

if so, what data describes the correlations between various causally disconnected domains.

Note added. While this manuscript was in its final stages of preparation, we learned of related work [42] on the conical geometries of sections 2 and 3.

\section{Rotational orbifolds and U-duality}

To begin, we consider the rotational orbifold $\left(A d S_{3} \times S^{3}\right) / \mathbb{Z}_{N}$. Our goal is to establish the relation of the orbifold geometry with a specific BPS state of the dual spacetime CFT. By U-duality, the BPS charges of the $n_{1}$ fundamental strings and $n_{5}$ fivebranes wrapped on $T^{4} \times S^{1}$ can be mapped to momentum and winding charges of a fundamental string on the $S^{1}$; the BPS configurations of such a string are wellknown to correspond to the different ways that one can satisfy the level-matching constraints by exciting left-moving oscillators while keeping the right-movers in their 
ground state. Lunin and Mathur [43, 11] have worked out the relation via U-duality of the metric sourced by such a fundamental string carrying winding and momentum on a circle (call it the $\tilde{x}_{5}$ direction) to the corresponding geometry of the D1-D5 or equivalently the F1-NS5 system; let us recall their analysis.

The chain of dualities in question is

$$
\text { F1-NS5 } \stackrel{T_{5}}{\longrightarrow} \text { P-NS5 } \stackrel{9 / 11 \text { flip }}{\longrightarrow} \text { P-D4 } \stackrel{T_{678}}{\longrightarrow} \text { P-D1 } \stackrel{S}{\longrightarrow} \text { P-F1 }
$$

(we assume that the NS5 brane is additionally wrapped on a $T^{4}$ along the 6789 directions). The BPS charges carried by the U-dual fundamental string are thus $n_{w}=n_{5}$ and $n_{p}=n_{1}$. As discussed in [44], the P-F1 duality frame is the appropriate low-energy description for the very-near horizon physics (i.e. ultra-low energy perturbations), and so it is appropriate to consider this U-duality frame for the purpose of understanding the structure of the vacuum and its low-lying excitations.

Level matching on the string requires a left-moving oscillator excitation of level $n_{1} n_{5}$. The general class of such states is (referring dimensions to the string scale $\ell_{s}$, which will be suppressed)

$$
\begin{aligned}
\tilde{x}_{i}(\tau+\sigma) & =\sum_{m} \frac{a_{m}}{\sqrt{m}} e^{i m(\tau+\sigma)}+c . c \\
\tilde{x}_{5} & =\frac{n_{1}}{\tilde{R}} \tau+n_{5} \tilde{R} \sigma \\
\tilde{t} & =E \tau
\end{aligned}
$$

where classically the amplitude of the oscillation is the square root of the occupation number $\left|a_{m}\right| \sim \sqrt{N_{m}}$. There are of order $\exp \left[2 \pi \sqrt{2 n_{1} n_{5}}\right]$ possible choices of oscillator excitation. The oscillator excitations may be classified according to representations of the symmetric group, and thus matched to the BPS states of the F1-NS5 or D1-D5 system, which are the orbifold cohomology classes of $\left(T^{4}\right)^{n_{1} n_{5}} / S_{n_{1} n_{5}}$ [45, 6].

The choice of oscillator excitation that is related to the $\left(A d S_{3} \times S^{3}\right) / \mathbb{Z}_{N}$ orbifold of $[19$ is

$$
\left(\alpha_{-N}\right)^{n_{1} n_{5} / N}
$$

where the polarization of the oscillators will be determined shortly. In particular, $N=1$ describes the $S L(2)$ invariant vacuum, or global $A d S$. We wish to see that the metric generated by this oscillator state U-dualizes to the orbifold metric [19

$$
\begin{aligned}
d s^{2} / \ell^{2}= & -\left(r^{2}+N^{-2}\right) d t^{2}+\left(r^{2}+N^{-2}\right)^{-1} d r^{2}+r^{2} d \phi^{2} \\
& +d \theta^{2}+\cos ^{2} \theta d \chi^{2}+\sin ^{2} \theta\left(d \psi-N^{-1} d \phi\right)^{2}
\end{aligned}
$$

This latter metric describes a state in the spacetime CFT with the quantum numbers (in the NS sector)

$$
\mathcal{L}_{0}+\frac{c}{24}=\frac{c}{12}\left(1-N^{-1}\right)=\mathcal{T}_{0}^{3}
$$

\footnotetext{
${ }^{5}$ Recall that in the F1-NS5 duality frame, the $A d S$ scale is $\ell=\sqrt{n_{5}} \ell_{s}$. In what follows, $A d S_{3} \times S^{3}$ coordinates will be made dimensionless by referring them to this scale.
} 
where $\mathcal{T}_{0}^{3}$ is the left-handed $S^{3}$ angular momentum, and $c=6 n_{1} n_{5}$. The rightmoving quantum numbers are identical.

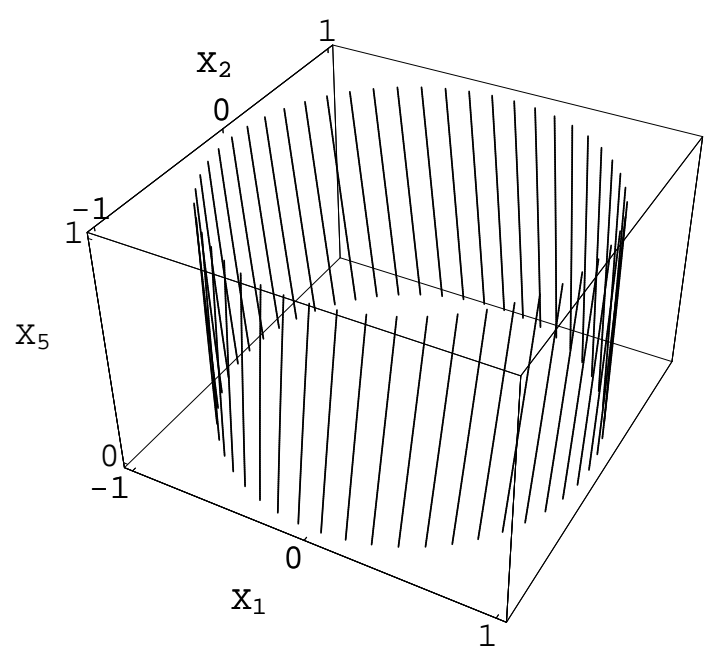

Figure 1: The spatial configuration of the U-dual string source for $n_{5}=50, N=1$, corresponding to global $A d S_{3} \times S^{3}$. The $\tilde{x}_{5}$ direction is periodically identified to make an $n_{5}$ times wound string. Smearing the source along $\tilde{x}_{5}$ generates a ring source in the $\tilde{x}_{1}-\tilde{x}_{2}$ plane.

The $S O(4)=S U(2) \times S U(2)$ angular momentum along the transverse directions 1234 in the system is $\mathcal{T}_{0}^{3}=\frac{1}{2}\left(M_{12}+M_{34}\right), \overline{\mathcal{T}}_{0}^{3}=\frac{1}{2}\left(M_{12}-M_{34}\right)$. When the F1NS5 system is mapped to the P-F1 duality frame, angular momentum $\left(-\frac{1}{2},-\frac{1}{2}\right)$ for each oscillator describes a string rotating in the 1-2 plane. The total angular momentum of the state (3) is thus $\mathcal{T}_{0}^{3}=\overline{\mathcal{T}}_{0}^{3}=n_{1} n_{5} / 2 N$ in $\mathrm{R}$ sector conventions, or $\frac{1}{2} n_{1} n_{5}(1-1 / N)$ in NS sector conventions (thus matching (5)). Eliminating $\tau$ and $\sigma$ from (2) (and solving the Virasoro constraints) gives the location in the target space of the string source:

$$
\begin{aligned}
& \tilde{x}_{1} \equiv \tilde{F}_{1}\left(\tilde{t}+\tilde{x}_{5}\right)=a \cos \left[\frac{N}{\tilde{R} n_{5}}\left(\tilde{t}+\tilde{x}_{5}\right)\right] \\
& \tilde{x}_{2} \equiv \tilde{F}_{2}\left(\tilde{t}+\tilde{x}_{5}\right)=a \sin \left[\frac{N}{\tilde{R} n_{5}}\left(\tilde{t}+\tilde{x}_{5}\right)\right]
\end{aligned}
$$

with the amplitude $a=\sqrt{n_{1} n_{5}} / N$. Plots of the spacetime configuration of such a source in the P-F1 duality frame are shown for global $A d S_{3} \times S^{3}, N=1$, in figure 1; and a naive configuration for $N>1$ is shown in in figure 2 . Note that the locus of the string in spacetime is traversed $N$ times as $\sigma$ goes from zero to $2 \pi$. 


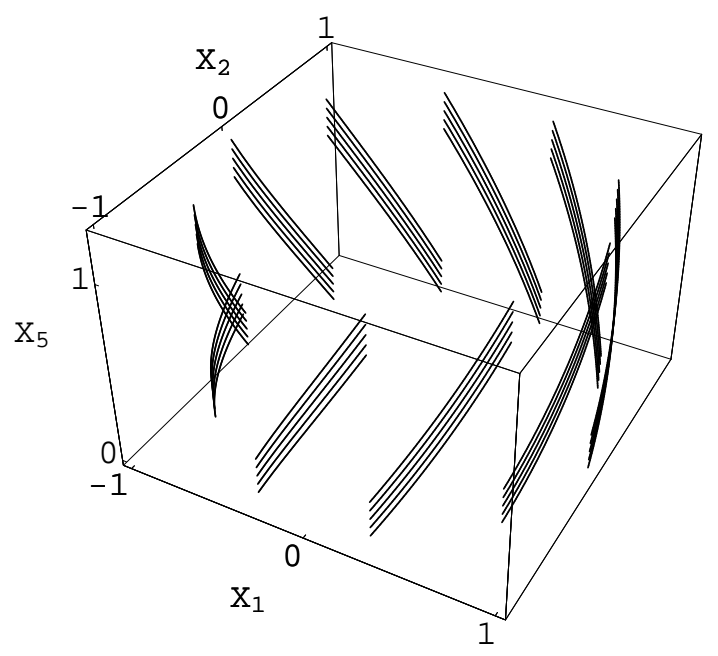

Figure 2: The spatial configuration of a $U$-dual string source for $n_{5}=50, N=$ 5 , related to $\left(A d S_{3} \times S^{3}\right) / \mathbb{Z}_{5}$. The strands are separated slightly for visualization purposes. The actual source for the orbifold puts the strands at finite separation.

The geometry sourced by this string configuration is

$$
\begin{aligned}
& d s^{2}=\tilde{H}^{-1}\left(-d \tilde{t}^{2}+d \tilde{x}_{5}^{2}+\tilde{K} d \tilde{v}^{2}+2 \tilde{A}_{i} d \tilde{x}_{i} d \tilde{v}\right)+d \tilde{x} \cdot d \tilde{x}+d y \cdot d y \\
& B_{\tilde{u} \tilde{v}}=-G_{\tilde{u} \tilde{v}}=\frac{1}{2} \tilde{H}^{-1}, \quad B_{\tilde{v} i}=-G_{\tilde{v} i}=-\tilde{H}^{-1} \tilde{A}_{i}, \quad e^{-2 \Phi}=\tilde{H}
\end{aligned}
$$

where $\tilde{H}, \tilde{K}$ are harmonic functions, $\vec{y}$ parametrizes the directions 6789 , and the field strength $F=d \tilde{A}$ satisfies $d^{*} F=0$, appropriate to the source (6):

$$
\tilde{H}=\frac{n_{5}}{|\tilde{x}-\tilde{F}|^{2}} \quad, \quad \tilde{K}=\frac{n_{5}\left|\tilde{F}^{\prime}\right|^{2}}{|\tilde{x}-\tilde{F}|^{2}} \quad, \quad \tilde{A}=-\frac{n_{5} \tilde{F}_{i}^{\prime}}{|\tilde{x}-\tilde{F}|^{2}}
$$

In order to be able to simply U-dualize this source configuration to the F1-NS5 duality frame, it is necessary to smear the source along $\tilde{x}_{5} \cdot{ }^{6}$ Defining

$$
\tilde{z}=\tilde{x}_{1}+i \tilde{x}_{2} \quad, \quad \tilde{w}=\tilde{x}_{3}+i \tilde{x}_{4}
$$

one finds after averaging the source over $\tilde{x}_{5}$ that

$$
\tilde{H}=\frac{n_{5}}{f_{0}}
$$

\footnotetext{
${ }^{6}$ The smearing operation is a linear operation on harmonic functions, hence preserves the property of solving the supergravity equations of motion.
} 


$$
\begin{aligned}
\tilde{K} & =\frac{n_{1}}{f_{0}} \\
\tilde{A}_{1} & =-\frac{\sqrt{n_{1} n_{5}} a y_{2}}{|\tilde{z}|^{2}} \frac{|\tilde{z}|^{2}+|\tilde{w}|^{2}+a^{2}-f_{0}}{f_{0}} \\
\tilde{A}_{2} & =\frac{\sqrt{n_{1} n_{5}} a y_{1}}{|\tilde{z}|^{2}} \frac{|\tilde{z}|^{2}+|\tilde{w}|^{2}+a^{2}-f_{0}}{f_{0}} \\
f_{0} & =\left[\left(|\tilde{z}|^{2}+|\tilde{w}|^{2}+a^{2}\right)^{2}-4 a^{2}|\tilde{z}|^{2}\right]^{1 / 2} .
\end{aligned}
$$

The solution is now ready for U-duality transformation to the F1-NS5 frame; the result is 43 , 11]

$$
\begin{aligned}
d s^{2} & =(H K)^{-1 / 2}\left[-(d t-A \cdot d x)^{2}+\left(d x_{5}+B \cdot d x\right)^{2}\right]+(H K)^{1 / 2} d x \cdot d x+d y \cdot d y \\
B_{t i} & =K^{-1} B_{i}, \quad B_{i 5}=-K^{-1} A_{i},
\end{aligned}
$$

where the F1-NS5 harmonic functions have the same form in terms of the dual (untilded) coordinates as the harmonics of the P-F1 solution $H(x)=\tilde{H}(\tilde{x}), K=\tilde{K}$, $A_{i}=\tilde{A}_{i}$; and $B_{i}$ is the dual gauge field of $A_{i}$ in the directions $1234, d B=-{ }^{*} d A$,

$$
\begin{aligned}
& B_{3}=-\frac{\sqrt{n_{1} n_{5}} a y_{4}}{|\tilde{w}|^{2}} \frac{|\tilde{z}|^{2}+|\tilde{w}|^{2}-a^{2}-f_{0}}{f_{0}} \\
& B_{4}=\frac{\sqrt{n_{1} n_{5}} a y_{3}}{|\tilde{w}|^{2}} \frac{|\tilde{z}|^{2}+|\tilde{w}|^{2}-a^{2}-f_{0}}{f_{0}}
\end{aligned}
$$

for more details, the reader may consult [43, 11].

One may readily verify that for $a=\sqrt{n_{1} n_{5}} / N$ one recovers the $\left(A d S_{3} \times S^{3}\right) / \mathbb{Z}_{N}$ orbifold geometry

$$
\begin{aligned}
d s^{2} / \ell^{2}=-\left(r^{2}\right. & \left.+\frac{a^{2}}{n_{1} n_{5}}\right) d t^{2}+r^{2} d x_{5}^{2}+\frac{d r^{2}}{r^{2}+\frac{a^{2}}{n_{1} n_{5}}} \\
& +\left(d \theta^{2}+\cos ^{2} \theta\left(d \psi-\frac{a}{\sqrt{n_{1} n_{5}}} d x_{5}\right)^{2}+\sin ^{2} \theta\left(d \chi-\frac{a}{\sqrt{n_{1} n_{5}}} d t\right)^{2}\right)
\end{aligned}
$$

after the change of variables

$$
\begin{aligned}
z & =\sqrt{n_{1} n_{5} r^{2}+a^{2}} \sin \theta e^{i \chi} \\
w & =\sqrt{n_{1} n_{5}} r \cos \theta e^{i \psi} \\
x_{5} & =\phi .
\end{aligned}
$$

The form of the metric in the subspace parametrized by $r, \theta, x_{5}$, and $\psi$ near the singularity at $r=0, \theta=\pi / 2$, is locally of the form $\mathbb{R}^{4} / \mathbb{Z}_{N}$ :

$$
d s_{4}^{2} \sim d(r / a)^{2}+d \theta^{2}+\frac{1}{N^{2}}(r / a)^{2} d x_{5}^{2}+(\theta-\pi / 2)^{2}\left(d \psi-\frac{1}{N} d x_{5}\right)^{2}
$$


(a standard form of the ALE metric at the orbifold point results from the substitution $r / a=\rho \sin \alpha, \theta-\pi / 2=\rho \cos \alpha)$. So indeed, $\left(A d S_{3} \times S^{3}\right) / \mathbb{Z}_{N}$ corresponds to the dual source (6).

Since the dual string source consists of $N$ strands which coincide in spacetime, the $N$-wound source can split into $N$ separate sources at no cost in energy. These $N$ strands each carry $n_{5} / N$ units of winding, and a fraction $n_{1} / N$ of the momentum?. The $N$ separate strands of P-F1 source yield a moduli space of multicenter solutions, obtained by replacing the harmonic functions and gauge fields (11) by their multicenter counterparts

$$
\begin{aligned}
\tilde{H} & =\frac{n_{5}}{N} \sum_{\alpha=1}^{N} \frac{1}{f_{0, \alpha}} \\
\tilde{K} & =\frac{n_{1}}{N} \sum_{\alpha=1}^{N} \frac{1}{f_{0, \alpha}} \\
\tilde{A}_{1} & =-\frac{\sqrt{n_{1} n_{5}} a}{N} \sum_{\alpha=1}^{N} \frac{\left(y_{2}-y_{2, \alpha}\right)}{\left|\tilde{z}-\tilde{z}_{\alpha}\right|^{2}} \frac{\left|\tilde{z}-\tilde{z}_{\alpha}\right|^{2}+\left|\tilde{w}-\tilde{w}_{\alpha}\right|^{2}+a^{2}-f_{0, \alpha}}{f_{0, \alpha}} \\
\tilde{A}_{2} & =\frac{\sqrt{n_{1} n_{5}} a}{N} \sum_{\alpha=1}^{N} \frac{\left(y_{1}-y_{1, \alpha}\right)}{\left|\tilde{z}-\tilde{z}_{\alpha}\right|^{2}} \frac{\left|\tilde{z}-\tilde{z}_{\alpha}\right|^{2}+\left|\tilde{w}-\tilde{w}_{\alpha}\right|^{2}+a^{2}-f_{0, \alpha}}{f_{0, \alpha}} \\
f_{0, \alpha} & =\left[\left(\left|\tilde{z}-\tilde{z}_{\alpha}\right|^{2}+\left|\tilde{w}-\tilde{w}_{\alpha}\right|^{2}+a^{2}\right)^{2}-4 a^{2}\left|\tilde{z}-\tilde{z}_{\alpha}\right|^{2}\right]^{1 / 2} \cdot
\end{aligned}
$$

One can show that, expanding the metric near the sources for separations $\left|z_{\alpha}\right|,\left|w_{\alpha}\right| \ll$ $a$, the metric assumes the ALE form

$$
\begin{aligned}
d s^{2} / \ell^{2} & =V^{-1}(\vec{x})\left(d x_{5}+\vec{\omega} \cdot d \vec{x}\right)^{2}+V(\vec{x}) d \vec{x} \cdot d \vec{x} \\
V(\vec{x}) & =\frac{\sqrt{n_{1} n_{5}}}{N a} \sum_{i=1}^{N}\left|\vec{x}-\vec{x}_{i}\right|^{-1}, \quad d V={ }^{*} d \omega ;
\end{aligned}
$$

here $\vec{x}$ is the three-vector of coordinates out of the directions 1234 that is locally transverse to the ring source. In other words, $V$ is the near-source limit of $(H K)^{1 / 2}$, and $\vec{\omega}$ is the near-source limit of $\vec{B}$ in the appropriate three directions. The fourth parameter of the multicenter solution is the separation of the sources from one another in the angular direction along the ring, or equivalently in the T-dual coordinate $\tilde{x}_{5}$. Upon smearing over $\tilde{x}_{5}$, these solutions separated along this angular direction are no different from the single center solutions (11); however, string dynamics on $\left(A d S_{3} \times S^{3}\right) / \mathbb{Z}_{N}$ knows about this deformation - for instance, in the analogous situation of string theory on $\mathbb{R}^{4} / \mathbb{Z}_{N}$, this deformation amounts to adjusting the NS

${ }^{7}$ N.B. It is not required that $N$ divides $n_{1}$ in the original F1-NS5 system; the quantization of the background string charge is not visible in the formalism of [14]. For the purposes of the present discussion, one can either distribute the momentum approximately equally among the $N$ separated strands, or assume that $N$ divides $n_{1}$ as well. 
$B$-flux through the $N-1$ collapsed cycles of the ALE space, while maintaining the conical metric of $\mathbb{R}^{4} / \mathbb{Z}_{N}$. Thus, the $\left(A d S_{3} \times S^{3}\right) / \mathbb{Z}_{N}$ orbifold spacetime yields a variation on the well-known duality between ALE spaces and NS fivebranes [46].

The couplings to the twisted sector marginal operators [19]

$$
\sum_{q}^{S L(2)} \sum_{N-q}^{S U(2)} \quad, \quad q=1, \ldots, N-1
$$

of the string theory orbifold $\left(A d S_{3} \times S^{3}\right) / \mathbb{Z}_{N}$ should therefore be identified with the moduli deformations of the multicenter solution (the four overall translation modes decouple in the near-horizon limit). Indeed, in the flat space limit $k \rightarrow \infty$ these operators become twisted moduli of the $\mathbb{R}^{4} / \mathbb{Z}_{N}$ orbifold CFT. The twist operators (18) lie in the $(2,2)$ representation of the global $S U(2) \times S U(2)$ R-symmetry, i.e. they are a vector in the $\mathbb{R}^{4}$ transverse to the fivebranes (the operators (18) are the bottom $(-,-)$ components of the multiplet). The operators (18) are products of parafermions from the $S L(2)$ and $S U(2)$ current algebra theories, and thus identical to the moduli of the $\left(\frac{S L(2, \mathbb{C})}{S U(2) \times \mathbb{R}} \times \frac{S U(2)}{U(1)}\right) / \mathbb{Z}_{N}$ sigma model of 47 describing a decoupling limit of slightly separated fivebranes; in the latter context, the deformations are the operators that move fivebranes in their transverse space (see [47] and section 1 below). In the fivebrane sigma model, it is known that at finite distance in the moduli space one finds singular worldsheet theories corresponding to points where two or more fivebranes come together. Therefore it is unreasonable that the fivebrane sources should coincide as in figure 2, since this should yield a singular worldsheet CFT.

The point described by the coset model has the fivebranes arranged in a $\mathbb{Z}_{N}$ symmetric fashion along a two-dimensional plane. This strongly suggests that we should interpret the magnetic $\mathbb{Z}_{N}$ symmetry of the orbifold theory in terms of a $\mathbb{Z}_{N}$ symmetric arrangement of the unsmeared sources around the ring of figure 2, see figure 3. The separation of the $N$ strands at the orbifold point is in a coordinate along which the source has been smeared, so the deformation along that direction will be invisible in the geometry, just as in the B-field deformation of $\mathbb{R}^{4} / \mathbb{Z}_{N}$.

In particular, we see that at finite distance in the moduli space, where strands of the dual P-F1 strings come together, the worldsheet CFT becomes singular. The point where all the strings come together is the configuration depicted in figure 2; we now see that this configuration lies at a singular point in the moduli space, where perturbative string theory breaks down. The fact that it takes finite energy above the $A d S$ vacuum to reach a singular CFT is consistent with the fact that the strands of the dual string are separated and have no moduli in the global case $N=1$ depicted in figure 2; it thus takes finite energy to push the fivebranes together. The scale of this energy $\mathcal{L}_{0} \sim O\left(n_{1}\right)$ is consistent with the fact that the separation of the strands scales as $\sqrt{n_{1}}$ (see the discussion below equation (2)), and so it takes an energy that scales as $n_{1}$ to push a pair of strands together. 


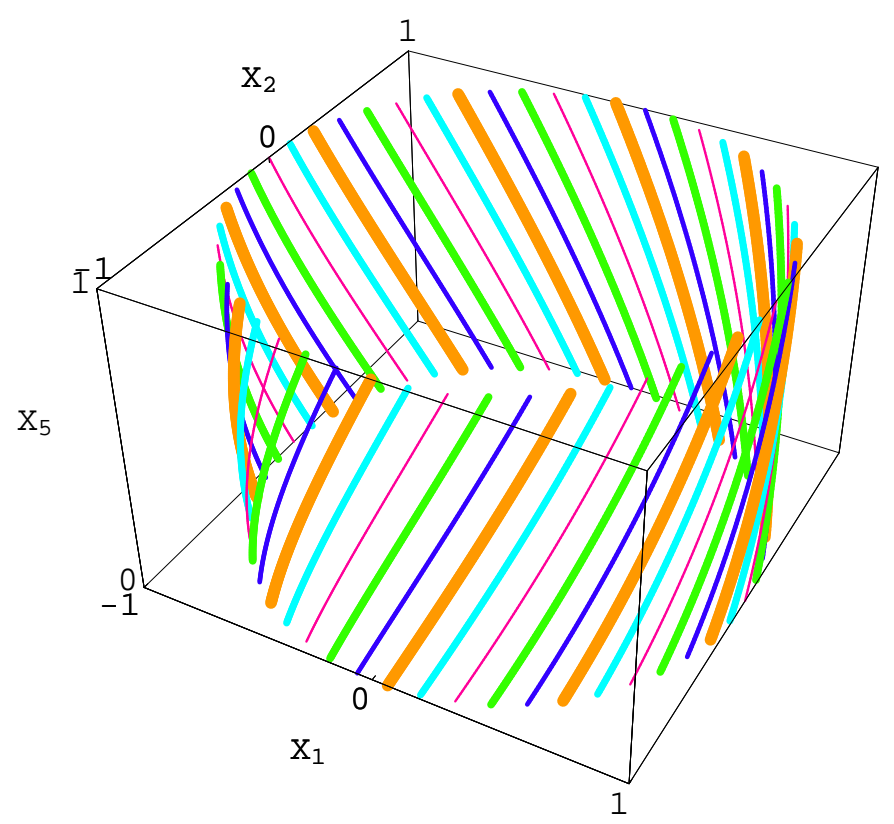

Figure 3: In the actual orbifold, the string source becomes $N$ strings arrayed in a $\mathbb{Z}_{N}$ symmetric fashion on a circle; here $n_{5}=50$, and $N=5$. The individual strings have been depicted with different colors and thicknesses for ease of visualization.

\section{Remarks:}

1.) It is important to note that, in the present context, U-duality and orbifolding do not commute. In the original F1-NS5 background, the effect of the orbifold is to transform the background from the $S L(2)$ invariant ground state of the spacetime CFT to a particular excited state

$$
\left(\sigma_{N}^{--}\right)^{n_{1} n_{5} / N}|0\rangle
$$

on the BPS line $\mathcal{L}_{0}+\frac{c}{24}=\mathcal{T}_{0}^{3}$. Carrying the $\mathbb{Z}_{N}$ symmetry that one wants to quotient by through the chain of dualities to the P-F1 frame, one finds that it acts there as an axial $\mathbb{Z}_{N}$ shift (since the $\tilde{x}_{5}$ circle is T-dual to the $x_{5}$ circle of the F1-NS5 frame) together with a $\mathbb{Z}_{N}$ rotation in the $\tilde{x}_{1}-\tilde{x}_{2}$ plane. Thus the result of such an orbifold in the P-F1 frame would produce a string sitting in a kind of Melvin

\footnotetext{
${ }^{8}$ We use the notation of the BPS states arising in the symmetric orbifold theory $\left(T^{4}\right)^{n_{1} n_{5}} / S_{n_{1} n_{5}}$, as twist ground states under the symmetric group. More generally, we can characterize the BPS states by the conjugacy class in the symmetric group $S_{n_{1} n_{5}}$ that they correspond to, and this sets up an unambiguous relation between the symmetric orbifold twist states and the BPS states of the F1-NS5 background with vanishing RR fields, the latter arising at a rather different point in the moduli space of the spacetime CFT [48].
} 
geometry, and not a transformed oscillator state in flat space, as is suggested by the above analysis.

2.) A related issue is the suggestion in [19], that there are new, nonperturbative kinds of orbifold constructions for $N \mid n_{1} n_{5}$ but $N \backslash n_{5}$. There are U-duality transformations that take $n_{1}, n_{5}$ to $n_{1}^{\prime}, n_{5}^{\prime}$ while keeping $n_{1} n_{5}=n_{1}^{\prime} n_{5}^{\prime}$, and such that $N \mid n_{5}^{\prime}$ but $N X n_{5}$ [48; the fact that there is a string theory orbifold in one U-duality frame but not the other suggested that there might be new kinds of orbifolds, hitherto unknown. We now understand that there is a BPS state (19) having the appropriate geometry $\left(A d S_{3} \times S^{3}\right) / \mathbb{Z}_{N}$, but it will not have any kind of standard orbifold construction, in the sense of having twisted sectors of the usual type, etc.

3.) Nevertheless, all P-F1 sources of the form (2) U-dualize (after smearing) into BPS geometries in the F1-NS5 frame that seem to be exact worldsheet CFT's. This is because all such geometries are 1/4 BPS, preserving eight supersymmetries. This amount of supersymmetry in spacetime is equivalent to $\mathcal{N}=(4,4)$ supersymmetry on the worldsheet; sigma models with this much supersymmetry are exact. In an orbifold CFT, the twisted sectors are an essential ingredient describing string theory on the geometry (13), in particular they resolve the singularity (provided there are no strong coupling singularities such as coincident fivebranes). Presumably there are states similarly localized near the singularity of the more general class of geometries associated to (2), for instance in the conical geometries of the previous remark; it would be very interesting to understand them, for instance their asymptotic density as discussed in 49, and their role in resolving the singularity in the geometry.

4.) The state that results from the orbifold operation is distinct from the corresponding BPS state of the $\mathcal{N}=4$ Liouville theory associated to AdS Chern-Simons supergravity, which is also associated to conical defects. As discussed for example in [50], the Liouville field is built (nonlocally) from the current sector of the spacetime CFT, and its Hilbert space has BPS states with the same quantum numbers as (19); however, these states are distinct. In particular, there is no way to distinguish different BPS states with the same quantum numbers $\mathcal{J}_{0}^{3}, \mathcal{L}_{0}$ via their properties under the $\mathcal{N}=4$ currents. We will elaborate upon the role of the Liouville field below.

To summarize, the orbifold operation in perturbative string theory maps global $A d S_{3} \times S^{3}$ to the geometry (13) of $\left(A d S_{3} \times S^{3}\right) / \mathbb{Z}_{N}$. We can then consider the image of this map under the duality between bulk and boundary theories under the AdS/CFT correspondence. The global geometry $A d S_{3} \times S^{3}$ maps to the $S L(2)$ invariant vacuum state of the dual CFT; correspondingly, the orbifold geometry $\left(A d S_{3} \times S^{3}\right) / \mathbb{Z}_{N}$ maps to the BPS state (19).

This correspondence has a fascinating reflection in the orbifold (spacetime) CFT $\left(T^{4}\right)^{n_{1} n_{5}} / S_{n_{1} n_{5}}$, which appears at a different point in the $\Gamma\left(n_{1} n_{5}\right) \backslash O(5,4) / O(5) \times$ $O(4)$ moduli space of the spacetime theory. Twist field correlation functions of

\footnotetext{
${ }^{9}$ See for instance [48] for a discussion of this moduli space, and in particular where the symmetric orbifold boundary theory $\left(T^{4}\right)^{n_{1} n_{5}} / S_{n_{1} n_{5}}$ and the perturbative bulk string theories of [14] are
} 
a free field orbifold CFT may be computed by a general procedure that employs branched coverings of the Riemann surface on which the CFT vertex operators are inserted [51, 52, 53]. The ramification points of the cover are the locations of the twist operator insertions. More recently, Lunin and Mathur [54, 55] have adapted this method to compute the correlation functions of the twist operators of the symmetric orbifold $\left(T^{4}\right)^{n_{1} n_{5}} / S_{n_{1} n_{5}}$. In this case, the ramification has the direct interpretation of sewing together the different copies of $T^{4}$ in the spacetime CFT, and there are remarkable simplifications in the structure of the correlation functions when one works on the covering space. For the particular case of the twist ground state (19), the two-point function corresponding to the propagation of this state is computed by passing to an $N$-fold branched cover, $w=z^{1 / N}$, of the CFT parameter space. Apart from the transformation of the measure (and a universal factor that implements the correct R-charge of the twist in the case of superconformal field theory), the covering space Riemann surface is the smooth two-sphere, and one is instructed to compute the vacuum partition function on the cover. The central charge $\tilde{c}$ of the covering space CFT is $1 / N$ of that of the original spacetime $\mathrm{CFT}$, $c=N \tilde{c}$, because of the $N$ copies of $T^{4} \mathrm{CFT}$ that have been sewn together in making the cover.

But this structure is exactly that of the perturbative string theory orbifold $\left(A d S_{3} \times S^{3}\right) / \mathbb{Z}_{N}$ - namely, there is an $N$-fold covering space which is smooth, i.e. the global $A d S_{3} \times S^{3}$ vacuum spacetime (see figure 4). For example, the relation $c=N \tilde{c}$ of the central charges was noted in [50]. It is standard to identify the parameter space of the CFT with the conformal boundary of AdS; the Euclidean continuation of $A d S_{3}$ to $H_{3}^{+}=S L(2, \mathbb{C}) / S U(2)$ has conformal boundary $S^{2}$, and the orbifold operation of the bulk theory on $H_{3}^{+}$directly generates the branched cover seen in the representations of the twist correlators of the boundary theory. Once again we see a rather precise correspondence between bulk and boundary theories. Of course, the perturbative bulk orbifold and the symmetric orbifold boundary theory are at different points in the moduli space of onebrane-fivebrane backgrounds, so one cannot compare directly; however, this remarkable parallel suggests to us that this property of covering onto smooth global $A d S$ may be protected by supersymmetry as one travels across the moduli space.

Although it would appear that the perturbative string orbifold procedure can be directly mapped to a corresponding representation in the exact boundary CFT dual, perhaps a note of caution is in order - the precise correspondence may be special to BPS states. In [50], a set of nonsupersymmetric orbifolds $\left(A d S_{3} / \mathbb{Z}_{N}\right) \times$ $S^{3}$ was also considered. In this case, $N$ can be arbitrarily large (in particular it need not divide $n_{1} n_{5}$ ). The perturbative spectrum of such orbifolds is tachyonic, indicating [50, 56] that they decay via the emission of a pulse of string excitations,

situated in it.

${ }^{10}$ This is related to the fact that in the long string sector of 18 there is an equivalence between a theory of central charge $6 n_{1} n_{5}$ on a circle of radius $R$ and a theory of central charge 6 on a circle of radius $n_{1} n_{5} R$. 


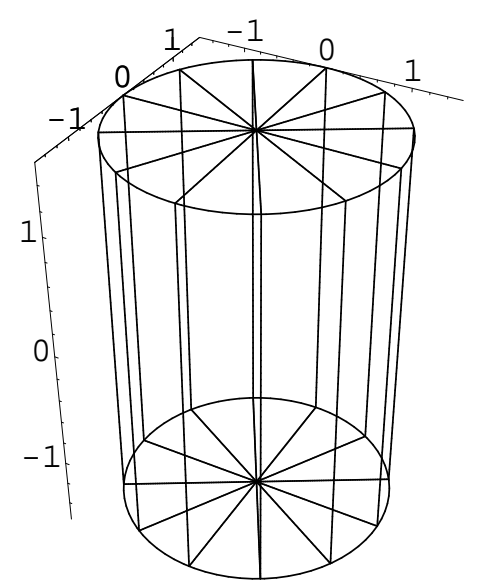

Figure 4: The $\left(A d S_{3} \times S^{3}\right) / \mathbb{Z}_{N}$ orbifold geometry covers smoothly onto global $A d S_{3} \times$ $S^{3}$. Here we have indicated the slicing of the global space into fundamental domains, with the quotient space being the conically singular geometry. This same covering procedure is involved in computations of twist operators in the $\left(T^{4}\right)^{n_{1} n_{5}} / S_{n_{1} n_{5}}$ orbifold related to the dual spacetime CFT.

eventually settling down to an ordinary excited state of $A d S_{3} \times S^{3}$ with the same quantum numbers. This state cannot have a naive covering space interpretation of the type considered above - the covering CFT would have central charge $\tilde{c}=$ $6 n_{1} n_{5} / N$, and $N$ can be arbitrarily large. Of course, the effect on the central charge is a special construction for twist operators of the symmetric group. There is certainly a covering interpretation for general $\mathbb{Z}_{N}$ twists in terms of a CFT of the same central charge as the original theory [51, 52, 53], but the path integral on the covering space is constrained - the fields must take on $\mathbb{Z}_{N}$ related values at $\mathbb{Z}_{N}$ related points on the covering space. It is only in the case of the symmetric orbifold twist operators that the covering space path integral is an unconstrained path integral [54, 55] in terms of a theory with central charge $N$ times smaller (i.e. $n_{1} n_{5} / N$ copies of the component CFT underlying the symmetric product). There might be a description of the $\left(A d S_{3} / \mathbb{Z}_{N}\right) \times S^{3}$ orbifold in terms of a twist operator in the dual CFT, of this more general type.

This description of BPS states and correlators in the spacetime CFT also sheds light on the role of the Liouville field that summarizes Chern-Simons $A d S_{3}$ supergravity [57]. The covering transformation yields a smooth Riemann surface from one with $\mathbb{Z}_{N}$ conical defects, but the corresponding coordinate map $w=z^{1 / N}$ affects the path integral via the conformal anomaly. As discussed for instance in [50], the Liouville field $\varphi_{L}=\log |\partial z / \partial w|^{2}$ summarizes the physical data of the Chern-Simons supergravity connection, in this instance the coordinate map relating flat space and 
the conical defect. Correspondingly, in [54, 55] this same Liouville field arises in the covering onto the vacuum spacetime via the conformal anomaly, and for the same reason - the gauge invariant modes of Chern-Simons supergravity can be identified with the modes of the current sector of the spacetime CFT.

\section{The excitation spectrum}

Having identified the state in the exact spacetime CFT corresponding to the perturbative $\left(A d S_{3} \times S^{3}\right) / \mathbb{Z}_{N}$ orbifold, we can now give a direct interpretation of the spectrum of low-energy perturbative string excitations around the orbifold. These consist of

- Untwisted short string states. These are characterized by their representation labels $\left(j, m, \bar{m} ; j^{\prime}, m^{\prime}, \bar{m}^{\prime}\right)$ under the worldsheet left and right $S L(2, \mathbb{R}) \times S U(2)$ current algebra, together with the oscillator content. The $S L(2)$ spin $j$ lies in the principal discrete series, $j \in \mathbb{R}, \frac{1}{2} \leq j<(k-1) / 2$. For massless (supergravity) states, the orbifold restricts roughly to states with $(m-\bar{m})-$ $\left(m^{\prime}-\bar{m}^{\prime}\right) \in N \mathbb{Z}$ (up to shifts due to the helicity content of the polarization state). In particular, excitations with $m=\bar{m}, m^{\prime}=\bar{m}^{\prime}$ are unaffected.

- Twisted short string states. These are roughly spanned by acting with twisted oscillators, and exponentials of the bosonized $J^{3}, J^{\prime 3}$ currents, on the $S L(2, \mathbb{R}) \times$ $S U(2)$ parafermion primaries (18). In the untwisted theory, the gap to the first oscillator excitations came from solving the mass shell condition

$$
\frac{-j(j-1)}{n_{5}}+\frac{j^{\prime}\left(j^{\prime}+1\right)}{n_{5}}+\mathbf{N}_{\mathrm{osc}}=\frac{1}{2}
$$

so the integer gap in oscillator number results in a gap in spacetime energy $\tilde{\mathcal{L}}_{0}$ of order $\delta j \sim \sqrt{n}_{5}$ in the measuring conventions of the covering space. In the orbifold theory, the fractional moding of twisted oscillators reduces the gap in the oscillator spectrum by a factor $N$ (again, as measured on the covering space), correspondingly the gap to the lowest stringy modes becomes $\delta \tilde{\mathcal{L}}_{0}=\delta j \sim \sqrt{n_{5}} / N$. In particular, for $N \sim n_{5}$, the gap becomes of the same order as that of the supergravity modes - there is no regime where supergravity is an effective approximation to the dynamics!

- Long string states. These states arise from principal continuous series representations [58] with $j=\frac{1}{2}+i s$, and have energy

$$
\tilde{\mathcal{L}}_{0}=\frac{n_{5} w}{4}+\frac{1}{w}\left(\frac{1+4 s^{2}}{4 n_{5}}+h_{\mathrm{int}}-\frac{1}{2}\right) .
$$

They describe strings that have escaped or are joining the background ensemble. Their coordinates are U-dual analogues of the twisted sector moduli 
separating fivebranes from the background ensemble. In the orbifold theory, fractional spectral flow by $w=p / N$ is allowed, provided one flows by an amount $-p / N$ in $S U(2)$; this means that the internal level is

$$
h_{\mathrm{int}}=\frac{j^{\prime}\left(j^{\prime}+1\right)}{4 n_{5}}-w m^{\prime}+\frac{n_{5}}{4} w^{2}+\mathbf{N}_{\mathrm{osc}} .
$$

Setting $m^{\prime}=0$ for simplicity, one sees that (after exciting a fermion oscillator $\mathbf{N}_{\text {osc }}=\frac{1}{2}$ to satisfy the GSO projection) that there are long string states at (covering space) energy

$$
\tilde{\mathcal{L}}_{0}=\frac{n_{5}}{N} p+\frac{N}{p n_{5}}\left(\frac{1+4 s^{2}}{4 n_{5}}+\frac{j^{\prime}\left(j^{\prime}+1\right)}{4 n_{5}}\right) .
$$

The gap to the emergence of long string states is thus also reduced by a factor of $N$; in particular, it is of the same order as the gap in supergravity modes for $N \sim n_{5}$.

The energy scales of these various excitations undergoes a further rescaling related to the orbifold identification. As discussed in [19], the time coordinate $t$ in terms of which the metric takes an asymptotic $A d S$ form, is rescaled by a factor of $N$ relative to the time coordinate $\tilde{t}$ of the covering space (unorbifolded global $A d S$ ), $t=N \tilde{t}$. Hence all energy scales $\mathcal{L}_{0}$ of the untwisted sector are rescaled by a factor of $1 / N$ with respect to the covering space energy $\tilde{\mathcal{L}}_{0}$,

$$
\mathcal{L}_{0}=\frac{1}{N} \tilde{\mathcal{L}}_{0}
$$

In particular, the gap in supergravity excitations is the inverse $A d S$ radius $\ell^{-1}$ in global $A d S$, and $(N \ell)^{-1}$ in the orbifold theory. In global $A d S$, the gap in the spacetime energy $m+\bar{m}$ and angular momentum $m-\bar{m}$ of the AdS modes of a given supergravity field (fixing also the quantum numbers on $S^{3}$ ) is accounted for by the fact that the modes are spanned by the action of $\mathcal{L}_{-1}, \overline{\mathcal{L}}_{-1}$ on the highest weight state:

$$
\Phi_{j m \bar{m}}^{S L(2)}=\left(\mathcal{L}_{-1}\right)^{m}\left(\overline{\mathcal{L}}_{-1}\right)^{\bar{m}} \Phi_{j 00}^{S L(2)}
$$

Thus the gap in energy is $1 / \ell$ (recall that spacetime energy is $\ell E=\mathcal{L}_{0}+\overline{\mathcal{L}}_{0}$ ). In the orbifold spacetime, $\mathcal{L}_{-1}$ descends from $\tilde{\mathcal{L}}_{-N}$ on the covering space, and does not span the full set of modes; thus, the $\Phi_{j m \bar{m}}^{S L(2)}$ are not related by the spacetime superconformal algebra. Shifting $m+\bar{m}$ by two (keeping $m-\bar{m}$ fixed) results in a change of the energy by an amount $2 / N \ell$.

This energy gap is inversely related to the return time of supergravity probes sent radially inward from the top of the throat in the full asymptotically flat D1-D5 geometry,

$$
\Delta t=\pi \frac{\sqrt{n_{1} n_{5}}}{a}
$$


as noted in [11]; for the orbifold backgrounds, one has $\Delta t=N \pi$. Our point here is that this time scale remains visible in the near-horizon limit, as the gap in the spectrum relative to the AdS scale. The effect of the orbifold is indeed to make a deeper gravitational well in the center of AdS, as expected from the fact that the conical defect starts to approach the black hole limit from below at large $N$. The closer one approaches the black hole threshold, the smaller the gap in the spectrum, due to the increasing redshift generated by the large mass at the center of $A d S$. At the extremal black hole point $\mathcal{L}_{0}=c / 12=\mathcal{T}_{0}^{3}$, the spectrum becomes continuous (in the classical theory).

We may interpret these various excitations in terms of the underlying brane dynamics as follows. The untwisted string states are related to the singlet sector - the overall $U(1)$ of the $U(N)$ gauge dynamics - of the fivebrane dual. This is in accord with the standard AdS/CFT duality between gauge invariant local operators and supergravity modes. The twisted sectors are the 'Cartan' multiplets describing individual groups of $n_{5} / N$ fivebranes (so one has the full set of Cartan modes for $N=n_{5}$ ), since the twisted sector moduli describe relative overall motion of the branes. The off-diagonal modes of fivebrane gauge dynamics are described as the fractional D-strings of the orbifold. Indeed, the separation of strands in the dual string source of global $A d S$ of figure $1, \sqrt{n_{1} / n_{5}} \ell_{s}$, corresponds (as it should) to the energy scale of D-branes in the F1-NS5 frame, since $g_{s}^{-1}=\sqrt{n_{1} / n_{5}}$. In the orbifold theory, the separation of the strands of the dual string decreases by a factor of $N$, reducing the energy cost of fractional D-strings in the F1-NS5 frame by a corresponding factor. Note that there are no noncompact spatial directions into which the RR flux of these fractional branes may escape; thus the fractional branes must always organize themselves into regular representation branes carrying no net fractional D1 charge; such branes can then disappear by leaving the fivebranes and decaying into untwisted string modes. This is a reflection of Gauss' law in the fivebrane gauge theory.

One should also note that the twisted moduli of the orbifold are effectively $1+1$ dimensional scalar fields, and so it is somewhat misleading to consider them as having expectation values as we have been doing. This is an artifact of the tree level approximation to the orbifold dynamics in perturbative string theory. At one loop, one will encounter an infrared divergence arising from the fluctuations of the moduli, and one will have to quantize them as collective coordinates (c.f. [59]). The moduli are thus the coordinates of a wavefunction for the fivebrane sources, whose understanding will require knowledge of the dynamics near the singular regions where fivebranes coincide.

It was noted in [19] that the gap to black hole states above the $\left(A d S_{3} \times S^{3}\right) / \mathbb{Z}_{N}$ orbifold is of order $n_{1} n_{5} / N^{2}$, which is of order $n_{1} / n_{5}=g_{6}^{2}$ when $N=n_{5} \boxplus$. There are two interesting interpretations of this scale. First, the energy to remove a fundamental string from the background ensemble is of order $n_{5}$; thus, the black hole threshold

\footnotetext{
${ }^{11} N$ is required to $n_{5}$ in order that the orbifold is not anomalous.
} 
is of order the energy required to remove all the strings from the background and put them on their Coulomb branch. Left behind would be a strong-coupling throat of the $n_{5}$ fivebranes, indicating the appearance of strong-coupling dynamics. Second, the energy cost of D-branes is of order $1 / g_{6}=\sqrt{n_{1} / n_{5}}$. The BTZ threshold is the energy cost of $n_{D} \sim \sqrt{n_{1} / n_{5}}$ D-branes, so that the effective gauge coupling $g_{6} n_{D} \sim 1$, and again the dynamics is strongly coupled. We regard these two estimates as yet another indication that the onset of black hole physics is a strong coupling phenomenon from the point of view of string dynamics.

\section{An aside on fivebranes}

The smearing of sources to obtain the geometry seen by low-energy string theory has applications to other backgrounds, in particular that of NS5-branes on the Coulomb branch studied in [47, 60]. There, it was claimed that the nonsingular coset CFT $\left(\frac{S L(2, \mathbb{C})}{S U(2) \times \mathbb{R}} \times \frac{S U(2)}{U(1)}\right) / \mathbb{Z}_{n_{5}} \mathbb{Z}$ describes the four-dimensional transverse space of separated fivebranes. On the other hand, supergravity would suggest that the target space is that of the multicenter CHS throat solution (directions along the fivebranes are suppressed)

$$
\begin{aligned}
d s^{2} & =-d t^{2}+H_{5}\left(|d z|^{2}+|d w|^{2}\right) \\
e^{2 \Phi} & =H_{5} \\
d B & ={ }^{*} d \Phi \\
H_{5} & =\sum_{\alpha=1}^{n_{5}} \frac{1}{\left|z-z_{\alpha}\right|^{2}+\left|w-w_{\alpha}\right|^{2}} .
\end{aligned}
$$

To see the relation between these two geometries, place the fivebranes at $z_{\alpha}=a \omega^{\alpha}$, $w_{\alpha}=0$ (where $\omega^{n_{5}}=1$ ), and smear the fields over the argument of $z$. One finds that $H_{5}$ is the same harmonic function $\tilde{H}$ of (16). After the change of variables (14), and defining $r / a=\sinh (\rho)$, the resulting geometry is

$$
\begin{aligned}
d s^{2} & =-d t^{2}+d \rho^{2}+d \theta^{2}+\frac{\sinh ^{2} \rho \cos ^{2} \theta}{\sinh ^{2} \rho+\cos ^{2} \theta} d \psi^{2}+\frac{\cosh ^{2} \rho \sin ^{2} \theta}{\sinh ^{2} \rho+\cos ^{2} \theta} d \phi^{2} \\
\Phi & =\log \left[\sinh ^{2} \rho+\cos ^{2} \theta\right] .
\end{aligned}
$$

Remarkably, this is the gometry of a coset CFT. A general class of Lorentzian coset models of the form $\frac{S L(2, \mathbb{R}) \times S U(2) \times U(1)}{U(1) \times U(1)}$, describing charged black holes, was considered in [61]. After a Wick rotation to Euclidean $A d S_{3}$, and taking the limit of the charge parameter $Q^{2} \rightarrow-1$ (in the conventions of [61]), one finds that the extra $U(1)$

\footnotetext{
${ }^{12}$ This cannot be reduced by a factor $N$ by the consideration of fractional brane representations because, as mentioned above, only regular representation branes are allowed.

${ }^{13}$ The Euclidean continuation of $S L(2, \mathbb{R})$ is the hyperbolic space $H_{3}^{+}=S L(2, \mathbb{C}) / S U(2)$. The $\mathbb{Z}_{n_{5}}$ identification is related to the GSO projection.
} 
decouples and the geometry becomes precisely that of (28)! Thus, smearing of the source provides the connection between the CHS geometry and the exact sigma model description of [47]. Note that smearing does not remove the singularities in the metric and dilaton; these still appear in (28), however they are apparently harmless in string theory, since the correlation functions of the coset are entirely well-behaved (at least at sufficiently low energy 47, 60]). This is a reflection of the absence of throat dynamics for separated fivebranes. Once again, the string theory remembers that the sources are localized and not smeared; for instance, D-strings stretch between specific $\mathbb{Z}_{N}$ symmetric points along the locus $\sinh ^{2} \rho+\cos ^{2} \theta=$ 0 62, 63.

\section{Comments on BTZ orbifolds, and cosmology}

In this section we try to apply some of the lessons learned above in the study of rotational orbifolds of $A d S_{3} \times S^{3}$ to other contexts, specifically the class of orbifolds that realize BTZ black holes as quotients of $A d S_{3}$ [20, 21].

\subsection{The BTZ geometry as an $A d S_{3}$ quotient}

Group elements $h \in S L(2, \mathbb{R})$ lie in one of three conjugacy classes - elliptic, parabolic, and hyperbolic, depending on whether $|\operatorname{Tr}[h]|$ is less than, equal to, or greater than two, respectively (the trace is in the two-dimensional representation). Elliptic elements are conjugate to a rotation, and lead to the class of conical defect spacetimes discussed above. The orbifold

$$
g \sim h g h \quad, \quad g \in S L(2, \mathbb{R})
$$

of $A d S_{3}$ by the action of the discrete group generated by a hyperbolic element $h$, leads to a fundamental domain of the identification which contains the BTZ black hole spacetime with zero angular momentum $(J=0)$ [20, 21] (the identification by a parabolic element yields the extremal $(\ell M=J)$ BTZ black hole). Let us parametrize $g \in S L(2, \mathbb{R})$ via the analogue of Euler angles

$$
\begin{aligned}
g & =e^{(t-\phi) i \sigma_{2} / 2} e^{\rho \sigma_{3}} e^{(t+\phi) i \sigma_{2} / 2} \equiv\left(\begin{array}{ll}
a & b \\
c & d
\end{array}\right) \\
& =\left(\begin{array}{cc}
\cos (t) \cosh (\rho)+\cos (\phi) \sinh (\rho) & \sin (t) \cosh (\rho)+\sin (\phi) \sinh (\rho) \\
-\sin (t) \cosh (\rho)+\sin (\phi) \sinh (\rho) & \cos (t) \cosh (\rho)-\cos (\phi) \sinh (\rho)
\end{array}\right) ;
\end{aligned}
$$

in these coordinates, the metric on $A d S_{3}$ takes the form

$$
d s_{A d S}^{2}=\ell^{2}\left(-\cosh ^{2} \rho d t^{2}+d \rho^{2}+\sinh ^{2} \rho d \phi^{2}\right) .
$$

The BTZ black hole geometry results from the identification (29), with $h=$ $\exp \left[\pi r_{+} \sigma_{3}\right]$; the mass of the BTZ black hole will then be $\ell M=\frac{1}{2} n_{1} n_{5} r_{+}^{2}$, with 


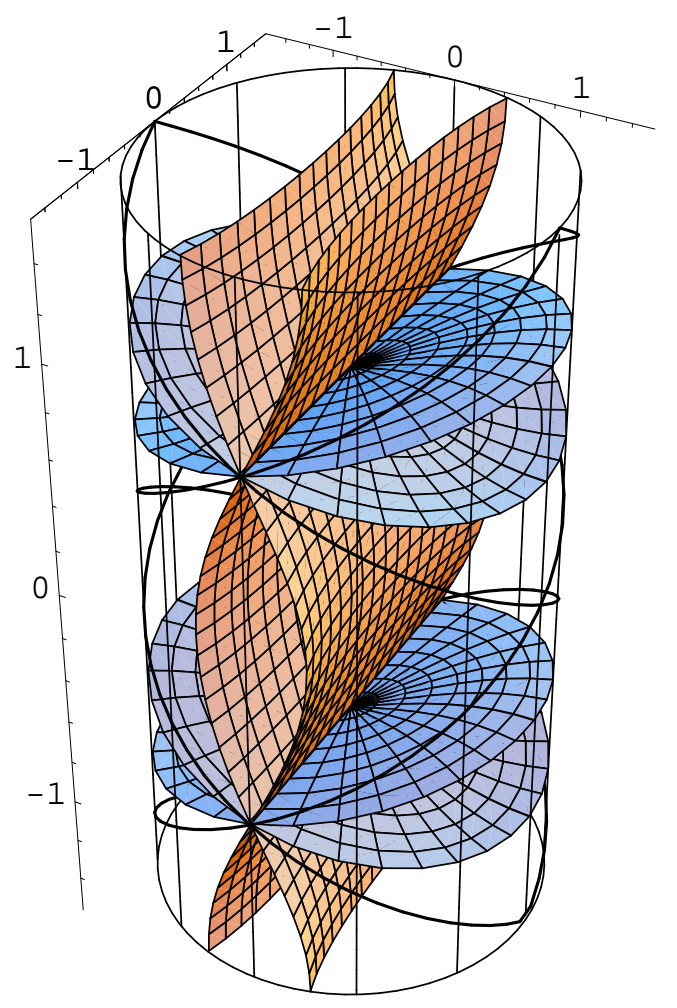

Figure 5: The fundamental domain of the identification of $A d S_{3}$ corresponding to a BTZ black hole. The vertical (amber) wavy surfaces are identified by a spacelike translation; also the horizontal (blue) disks are identified by a timelike translation. The identification has surfaces of null identification which intersect the boundary along the thick (black) helices. The intersection of all these surfaces is the spacelike BTZ black hole singularity.

$r_{+}$the horizon radius in units of the $A d S$ curvature scale $\ell$. The fundamental domain of this orbifold is depicted in figure 5 , with the radial coordinate redefined as $\sinh [\rho]=\tan [\theta]$ in order to view $A d S_{3}$ as the canonical solid cylinder. Slices through the $A d S$ cylinder at $\sin \phi=0$ and $\cos \phi=0$ are depicted in figure 6; the slice $\sin \phi=0$ exhibits the identification, while the slice $\cos \phi=0$ reveals the standard Penrose diagram of the Kruskal extension of the black hole geometry.

For completeness, we also give the metric geometry that results from the quotient, and its relation to the group manifold, following [20, 21]. In the standard BTZ coordinates, the black hole metric takes the form

$$
d s_{B T Z}^{2} / \ell^{2}=-\left(\hat{r}^{2}-r_{+}^{2}\right) d \hat{t}^{2}+\left(\hat{r}^{2}-r_{+}^{2}\right)^{-1} d \hat{r}^{2}+\hat{r}^{2} d \hat{\phi}^{2}
$$




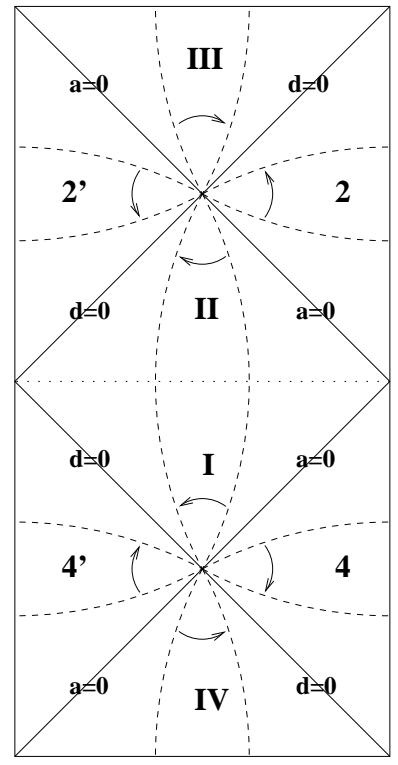

$\sin (\phi)=0$

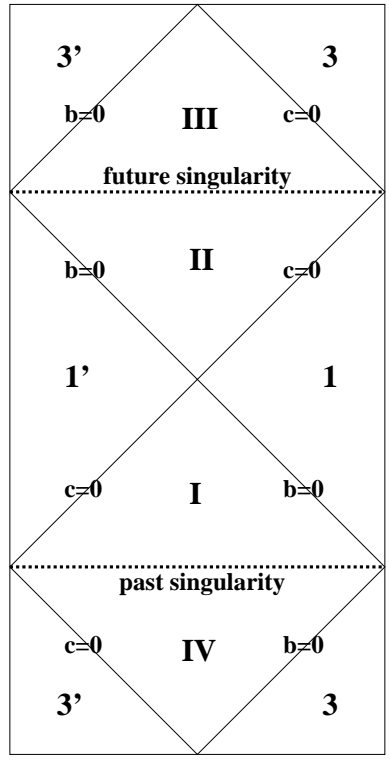

$\cos (\phi)=0$

Figure 6: Slices of the geometry of figure 5, at $\sin \phi=0$ and at $\cos \phi=0$. The slice $\sin \phi=0$ exhibits the identification of global AdS involved, while the slice $\cos \phi=0$ shows the Penrose diagram. The labels of the various regions are adopted from the conventions of $[49$. Also, the $2 \times 2$ matrix element of $g$ which vanishes along various null curves is indicated.

with $\hat{\phi} \sim \hat{\phi}+2 \pi$. The relation to the global coordinates (31) is best established in the Kruskal extension of (32)

$$
u=\sqrt{\frac{\hat{r}-r_{+}}{\hat{r}+r_{+}}} \exp \left[+r_{+} \hat{t}\right] \quad, \quad v=\sqrt{\frac{\hat{r}-r_{+}}{\hat{r}+r_{+}}} \exp \left[-r_{+} \hat{t}\right],
$$

for which the metric is

$$
d s_{B T Z}^{2} / \ell^{2}=(1-u v)^{-2}\left[4 d u d v+r_{+}^{2}(1+u v)^{2} d \hat{\phi}^{2}\right] .
$$

The black hole horizon is the surface $u v=0$, the singularities are at $u v=-1$, and the asymptotically locally $A d S$ boundary is at $u v=+1$. Near the singularity, the geometry is like that of a Milne universe as the $\hat{\phi}$ circle shrinks to zero size. In these coordinates, the $S L(2, \mathbb{R})$ matrix $g=\left(\begin{array}{ll}a & b \\ c & d\end{array}\right)$ of $(30)$ is written

$$
a=\left(\frac{\hat{r}}{r_{+}}\right) \exp \left[+r_{+} \hat{\phi}\right]=\left(\frac{1+u v}{1-u v}\right) \exp \left[+r_{+} \hat{\phi}\right]
$$




$$
\begin{aligned}
b & =\left(\frac{\hat{r}^{2}}{r_{+}^{2}}-1\right)^{1 / 2} \exp \left[+r_{+} \hat{t}\right]=\frac{2 u}{1-u v} \\
c & =\left(\frac{\hat{r}^{2}}{r_{+}^{2}}-1\right)^{1 / 2} \exp \left[-r_{+} \hat{t}\right]=\frac{2 v}{1-u v} \\
d & =\left(\frac{\hat{r}}{r_{+}}\right) \exp \left[-r_{+} \hat{\phi}\right]=\left(\frac{1+u v}{1-u v}\right) \exp \left[-r_{+} \hat{\phi}\right]
\end{aligned}
$$

(recall the identification is $\hat{\phi} \sim \hat{\phi}+2 \pi$ ). This parametrization covers regions $1,1^{\prime}, I, I I$ in figure 6 ; the same parametrization covers regions $3,3^{\prime}, I I I, I V$ if we send $g \rightarrow-g$. The regions $2,2^{\prime}$ are covered by

$$
\begin{aligned}
& a=\sinh \left[\rho^{\prime}\right] e^{t^{\prime}}, \quad b=\cosh \left[\rho^{\prime}\right] e^{\phi^{\prime}} \\
& c=-\cosh \left[\rho^{\prime}\right] e^{-\phi^{\prime}}, \quad d=-\sinh \left[\rho^{\prime}\right] e^{-t^{\prime}}
\end{aligned}
$$

(and similarly regions $4,4^{\prime}$ by sending $g \rightarrow-g$ ); the metric in these regions is

$$
d s^{2}=\ell^{2}\left(-\sinh ^{2} \rho^{\prime} d t^{\prime 2}+d \rho^{\prime 2}+\cosh ^{2} \rho^{\prime} d \phi^{\prime 2}\right) .
$$

The action of the identification in these regions is $t^{\prime} \sim t^{\prime}+2 \pi r_{+}$, and thus has a singularity at $\rho^{\prime}=0$. This metric is identical to (32) (via $\hat{r} / r_{+}=\cosh \rho^{\prime}, r_{+} \hat{t}=t^{\prime}$, $r_{+} \hat{\phi}=\phi^{\prime}$ ), except that the temporal coordinate is periodically identified rather than the spatial coordinate.

\subsection{Is there an exact orbifold description?}

There have been several attempts to give meaning to perturbative string theory on (29) and similar time-dependent orbifolds [26, 27, 30, 31, 33, 35, 36, 37, 38, 64, 39, 40, 65] Certainly, if string perturbation theory is sensible on this orbifold one will reap valuable information about the AdS/CFT correspondence. Furthermore, near the singularity the geometry is locally that of identification of Minkowski space under a discrete boost - the Milne spacetime - that has figured in several recent discussions of string cosmology [25, 32, 34, 36, 37, 38, 39, 65, and is a prototypical example of a pre-big bang cosmological scenario (c.f. 66] for a review). In addition to the standard Kruskal extension of the black hole, which is the fundamental domain of the region where the identification is spacelike, there are also regions of timelike identification, see figure 5.

Naively, the effect of the orbifold procedure might be expected to be the identification of the parameter space cylinder of the spacetime CFT by the action of (29) on the corresponding conformal boundary of $S L(2, \mathbb{R})$. If so, then the problem would reduce to understanding $2 \mathrm{~d}$ field theory in the cyclic Milne-like cosmological spacetime in which the dual 'boundary' CFT lives. On the boundary, the BTZ and global coordinates are related by

$$
\exp \left[ \pm r_{+}(\hat{t} \pm \hat{\phi})\right]= \pm \tan [(t \pm \phi) / 2]
$$


and the identification is $\hat{\phi} \sim \hat{\phi}+2 \pi$.

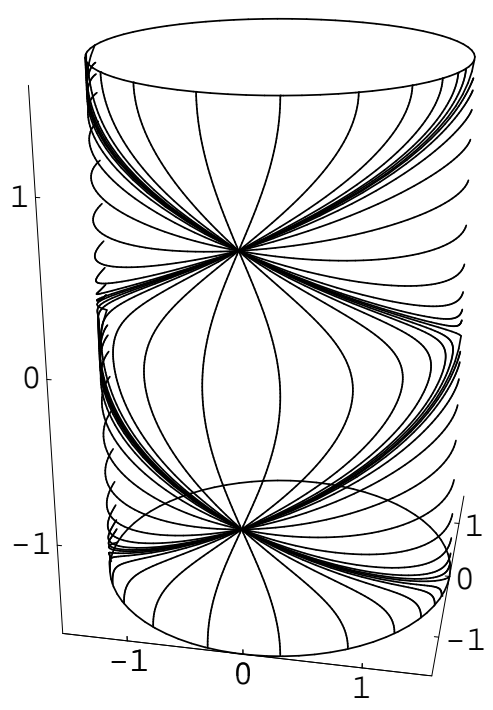

Figure 7: Covering of the BTZ spacetime onto global $A d S_{3}$. The curves where the boundaries of the fundamental domain intersect the boundary of $A d S_{3}$ are indicated for half of the boundary. The points of intersection of all these curves ought to be the locations of twist operators in the spacetime CFT.

We have seen that the effect of a quotient of the bulk theory by an elliptic $\mathbb{Z}_{N}$ transformation amounts to consideration of a different state (19) in the dual CFT, whose correlators are indeed computed by a $\mathbb{Z}_{N}$ covering of the Riemann surface on which they are defined, see figure 4. For the orbifold (29) related to the BTZ geometry, the slicing of global $A d S_{3}$ into fundamental domains under the identification is depicted in figure 7 .

The idea we wish to entertain is that the Milne-type BTZ singularity is described in the exact dual spacetime CFT as a 'vertex operator' that lives at and implements the big crunch/big bang on the boundary. This picture is somewhat different from that of [12], where it was proposed to describe the black hole as a correlated state in the boundary theory (see also [28, 67, 68] for related discussions). These descriptions employ the BTZ time of the static asymptotic observer and therefore can't see the big crunch/big bang singularity, since this occurs at infinite time in those coordinates. The orbifold description is an attempt to maintain the use of the global time, which is

\footnotetext{
${ }^{14}$ It is worth noting that the boundary structure of the identification is little changed upon incorporation of $A d S_{3}$ angular momentum in the geometry. The argument of the exponential on the LHS of (38) is simply modified to $(\hat{t} \pm \hat{\phi})\left(r_{+} \mp r_{-}\right)$, even though in the interior there is a rather dramatic modification of the Penrose diagram and causal structure, (c.f. 20, 21) - an inner horizon develops, and the singularity becomes timelike rather than spacelike.
} 
more akin to the proper time of an infalling observer, especially near the singularity.

The identification $\hat{\phi} \sim \hat{\phi}+2 \pi$ of the BTZ orbifold implies that the corresponding CFT state is not the CFT vacuum. The appropriate state in the boundary CFT is the one that yields the proper BTZ correlators, for instance the two-point function 70 , 71, 12

$$
\begin{aligned}
&\left\langle\mathcal{O}\left(\hat{x}_{+}, \hat{x}_{-}\right) \mathcal{O}\left(\hat{x}_{+}^{\prime}, \hat{x}_{-}^{\prime}\right)\right\rangle \sim \\
& \sum_{m=-\infty}^{\infty}\left[\sinh \left[\frac{r_{+}}{2}\left(\hat{x}_{+}-\hat{x}_{+}^{\prime}+2 \pi m\right)\right] \sinh \left(\frac{r_{+}}{2}\left(\hat{x}_{-}-\hat{x}_{-}^{\prime}+2 \pi m\right)\right]\right]^{-2 h}
\end{aligned}
$$

between operators on the same Kruskal boundary; here $\hat{x}_{ \pm}=\hat{t} \pm \hat{\phi}$ and $h$ is the conformal weight. As usual, the thermal nature of this state with respect to static observers in the (hatted) black hole coordinates is reflected in the invariance of this expression under the transformation $\hat{t} \rightarrow \hat{t}+2 \pi i / r_{+}$, which is an invariance of the global coordinates, c.f. (38). Mapping back to the global coordinates via (38) yields (with similarly $x_{ \pm}=t \pm \phi$ )

$$
\left\langle\mathcal{O}\left(x_{+}, x_{-}\right) \mathcal{O}\left(x_{+}^{\prime}, x_{-}^{\prime}\right)\right\rangle \sim \sum_{m=-\infty}^{\infty}\left[f_{m}\left(x_{+}, x_{+}^{\prime}\right) \times f_{m}\left(x_{-}, x_{-}^{\prime}\right)\right]^{-h},
$$

where $f_{m}\left(x, x^{\prime}\right)$ has the form

$$
f_{m}\left(x, x^{\prime}\right)=\left(e^{m \pi r_{+}} \sin ^{2} \frac{x}{2} \cos ^{2} \frac{x^{\prime}}{2}+e^{-m \pi r_{+}} \cos ^{2} \frac{x}{2} \sin ^{2} \frac{x^{\prime}}{2}-\frac{1}{2} \sin x \sin x^{\prime}\right) .
$$

The expression in brackets in the $m=0$ term of this sum reduces to the form $\sin ^{2}\left[\frac{1}{2}\left(x_{+}-x_{+}^{\prime}\right)\right] \sin ^{2}\left[\frac{1}{2}\left(x_{-}-x_{-}^{\prime}\right)\right]$, which is the appropriate correlation function of scaling operators (in the global coordinates) in the $S L(2, \mathbb{R})$ invariant vacuum; the remaining terms may then be interpreted as coming from the identification that yields the BTZ state. Note that, as discussed above for the case of rotational orbifolds, the vacuum path integral on the covering space is not the right result the fields take on the same values at points related by the group identification. 10 The relation of $1+1 \mathrm{~d}$ scalar field modes respecting identification under a boost, and the standard global modes for a free scalar field, has been explored in [38] (see also [39]).

The analogue of the two-point function (40) for dimension $(1,0)$ fields can be used to compute the stress tensor of the black hole state, by taking the coincidence limit and subtracting the pole term; one finds (up to an additive constant)

$$
T_{++} \sim\left(2 \sum_{m=1}^{\infty} \frac{1}{\sinh ^{2} m \pi r_{+}}\right) \frac{1}{\sin ^{2} x_{+}},
$$

\footnotetext{
${ }^{15}$ Although apparently absent this identification the appropriate state is the CFT vacuum; for a discussion in a closely related context of vacua related to the coordinate transformation (38), see for instance [69].

${ }^{16}$ And thus we see that (as remarked above) if it were not for this additional restriction, the eternal black hole and the global $A d S$ vacuum would be the same state, c.f. [69].
} 
indicating that the energy of the black hole state is infinite due to a rather bad divergence of the stress-energy along the light cones emananating from the singular point of the identification. This result is consistent with the known divergence of the bulk stress tensor at the fixed point of the identification or on the inner horizon of the spinning BTZ black hole [72]. This strongly divergent stress-energy may be an indication that the orbifold interpretation of the BTZ state is not viable.

There are regions of timelike identification in figure 5. The large radius boundary of the fundamental domain of the identification consists of two spacelike and two timelike cylinders which meet at the points where the BTZ singularity intersects the $A d S$ boundary; the spacelike and timelike cylinders on the boundary are roughly speaking interchanged by the operation $t \leftrightarrow \phi$. These regions of timelike identification are analogues of the 'whiskers' of the closely related Nappi-Witten cosmological spacetime [73 discussed recently in 40 (indeed, the labelling of the slices in figure 6 is adopted from [40]). Roughly, the gauging performed in the Nappi-Witten geometry removes the BTZ radial direction from the Kruskal region of the global geometry, and the angular direction from the 'whisker' region. In [40] it was argued that observables are naturally defined in these regions, in contrast to the conventional view (c.f. [74, 21]) that such regions are somehow forbidden or unstable. These regions are certainly present if we regard the BTZ geometry as an orbifold of global $A d S_{3}$. In a sense, these regions are built-in by the identification and the nature of the field configurations contributing to the path integral; we see no obvious reason to exclude them. Again, making sense of them (or not) in the $1+1$ dimensional boundary CFT will go a long way toward settling the question of their permissibility in a quantum theory of gravity.

Acknowledgments: We thank D. Kutasov, J. Maldacena, and S. Mathur for discussions; EM wishes to thank LPTHE, Université Pierre et Marie Curie, for its hospitality during the completion of the manuscript. This work was supported by DOE grant DE-FG02-90ER-40560. 


\section{References}

[1] A. Strominger and C. Vafa, "Microscopic origin of the Bekenstein-Hawking entropy," Phys. Lett. B379 (1996) 99-104, http://arXiv.org/abs/hep-th/9601029.

[2] C. G. Callan and J. M. Maldacena, "D-brane approach to black hole quantum mechanics," Nucl. Phys. B472 (1996) 591-610, http://arXiv.org/abs/hep-th/9602043.

[3] S. R. Das and S. D. Mathur, "Comparing decay rates for black holes and D-branes," Nucl. Phys. B478 (1996) 561-576, http://arXiv.org/abs/hep-th/9606185.

[4] L. Susskind and E. Witten, "The holographic bound in anti-de Sitter space," http://arXiv.org/abs/hep-th/9805114.

[5] E. Witten, "Anti-de Sitter space, thermal phase transition, and confinement in gauge theories," Adv. Theor. Math. Phys. 2 (1998) 505-532, http://arXiv.org/abs/hep-th/9803131.

[6] J. M. Maldacena and A. Strominger, "AdS(3) black holes and a stringy exclusion principle," JHEP 12 (1998) 005, http://arXiv.org/abs/hep-th/9804085.

[7] R. Dijkgraaf, J. M. Maldacena, G. W. Moore, and E. Verlinde, "A black hole Farey tail," http://arXiv.org/abs/hep-th/0005003.

[8] J. M. Maldacena, "The large $N$ limit of superconformal field theories and supergravity," Adv. Theor. Math. Phys. 2 (1998) 231-252, http://arXiv.org/abs/hep-th/9711200.

[9] G. T. Horowitz and D. Marolf, "Where is the information stored in black holes?," Phys. Rev. D55 (1997) 3654-3663, http://arXiv.org/abs/hep-th/9610171.

[10] T. Banks, M. R. Douglas, G. T. Horowitz, and E. J. Martinec, "AdS dynamics from conformal field theory," http://arXiv.org/abs/hep-th/9808016.

[11] O. Lunin and S. D. Mathur, "AdS/CFT duality and the black hole information paradox," Nucl. Phys. B623 (2002) 342-394, http://arXiv.org/abs/hep-th/0109154.

[12] J. M. Maldacena, "Eternal black holes in anti-de Sitter," http://arXiv.org/abs/hep-th/0106112.

[13] N. Seiberg and E. Witten, "The D1/D5 system and singular CFT," JHEP 04 (1999) 017, http://arXiv.org/abs/hep-th/9903224. 
[14] A. Giveon, D. Kutasov, and N. Seiberg, "Comments on string theory on AdS(3)," Adv. Theor. Math. Phys. 2 (1998) 733-780, hep-th/9806194.

[15] R. Dijkgraaf, E. Verlinde, and H. Verlinde, "5d black holes and matrix strings," Nucl. Phys. B506 (1997) 121-142, http://arXiv.org/abs/hep-th/9704018.

[16] E. Witten, "On the conformal field theory of the Higgs branch," JHEP 07 (1997) 003, http://arXiv.org/abs/hep-th/9707093.

[17] R. Dijkgraaf, "Instanton strings and hyperkaehler geometry," Nucl. Phys. B543 (1999) 545-571, http://arXiv.org/abs/hep-th/9810210.

[18] J. M. Maldacena and L. Susskind, "D-branes and fat black holes," Nucl. Phys. B475 (1996) 679-690, http://arXiv.org/abs/hep-th/9604042.

[19] E. J. Martinec and W. McElgin, "String theory on AdS orbifolds," JHEP 04 (2002) 029, http://arXiv.org/abs/hep-th/0106171.

[20] M. Banados, C. Teitelboim, and J. Zanelli, "The black hole in three-dimensional space-time," Phys. Rev. Lett. 69 (1992) 1849-1851, http://arXiv.org/abs/hep-th/9204099.

[21] M. Banados, M. Henneaux, C. Teitelboim, and J. Zanelli, "Geometry of the $(2+1)$ black hole," Phys. Rev. D48 (1993) 1506-1525, http://arXiv.org/abs/gr-qc/9302012.

[22] D. Kutasov, F. Larsen, and R. G. Leigh, "String theory in magnetic monopole backgrounds," Nucl. Phys. B550 (1999) 183-213, http://arXiv.org/abs/hep-th/9812027.

[23] F. Larsen and E. J. Martinec, "Currents and moduli in the $(4,0)$ theory," JHEP 11 (1999) 002, http://arXiv.org/abs/hep-th/9909088.

[24] J. M. Maldacena, J. Michelson, and A. Strominger, "Anti-de Sitter fragmentation," JHEP 02 (1999) 011, http://arXiv.org/abs/hep-th/9812073.

[25] G. T. Horowitz and A. R. Steif, "Singular string solutions with nonsingular initial data," Phys. Lett. B258 (1991) 91-96.

[26] Y. Satoh, "Study of three dimensional quantum black holes," http://arXiv.org/abs/hep-th/9705209.

[27] Y. Satoh, "Ghost-free and modular invariant spectra of a string in $\mathrm{SL}(2, \mathrm{R})$ and three-dimensional black hole geometry," Nucl. Phys. B513 (1998) 213-228, http://arXiv.org/abs/hep-th/9705208. 
[28] G. T. Horowitz and D. Marolf, "A new approach to string cosmology" JHEP 07 (1998) 014, http://arXiv.org/abs/hep-th/9805207.

[29] S. Aminneborg, I. Bengtsson, D. Brill, S. Holst, and P. Peldan, "Black holes and wormholes in 2+1 dimensions," Class. Quant. Grav. 15 (1998) 627-644, http://arXiv.org/abs/gr-qc/9707036.

[30] K. Behrndt, "Orbifolds of $\operatorname{AdS}(3)$ and fixpoints of the CFT," Fortsch. Phys. 48 (2000) 15-18, http://arXiv.org/abs/hep-th/9812169.

[31] K. Behrndt and D. Lust, "Branes, waves and AdS orbifolds," JHEP 07 (1999) 019, http://arXiv.org/abs/hep-th/9905180.

[32] J. Khoury, B. A. Ovrut, N. Seiberg, P. J. Steinhardt, and N. Turok, "From big crunch to big bang," Phys. Rev. D65 (2002) 086007, http://arXiv.org/abs/hep-th/0108187.

[33] S. Hemming and E. Keski-Vakkuri, "The spectrum of strings on BTZ black holes and spectral flow in the SL(2,R) WZW model," Nucl. Phys. B626 (2002) 363-376, http://arXiv.org/abs/hep-th/0110252.

[34] N. Seiberg, "From big crunch to big bang - is it possible?," http://arXiv.org/abs/hep-th/0201039.

[35] L. Cornalba and M. S. Costa, "A new cosmological scenario in string theory," http://arXiv.org/abs/hep-th/0203031.

[36] N. A. Nekrasov, "Milne universe, tachyons, and quantum group," http://arXiv.org/abs/hep-th/0203112.

[37] J. Simon, "The geometry of null rotation identifications," http://arXiv.org/abs/hep-th/0203201.

[38] A. J. Tolley and N. Turok, "Quantum fields in a big crunch / big bang spacetime," http://arXiv.org/abs/hep-th/0204091.

[39] H. Liu, G. Moore, and N. Seiberg, "Strings in a time-dependent orbifold," http://arXiv.org/abs/hep-th/0204168.

[40] S. Elitzur, A. Giveon, D. Kutasov, and E. Rabinovici, "From big bang to big crunch and beyond," http://arXiv.org/abs/hep-th/0204189.

[41] E. E. Flanagan, "Quantum mechanical instabilities of Cauchy horizons in two dimensions: A modified form of the blueshift instability mechanism," http://arXiv.org/abs/gr-qc/9711066. 
[42] O. Lunin and S. D. Mathur, "Rotating deformations of $A d S \times S^{3}$, the orbifold CFT and strings in the pp-wave limit," http://arXiv.org/abs/hep-th/0206107.

[43] O. Lunin and S. D. Mathur, "Metric of the multiply wound rotating string," Nucl. Phys. B610 (2001) 49-76, http://arXiv.org/abs/hep-th/0105136.

[44] E. Martinec and V. Sahakian, "Black holes and five-brane thermodynamics," Phys. Rev. D60 (1999) 064002, hep-th/9901135.

[45] C. Vafa, "Gas of D-branes and Hagedorn density of BPS states," Nucl. Phys. B463 (1996) 415-419, http://arXiv.org/abs/hep-th/9511088.

[46] D. Kutasov, "Orbifolds and solitons," Phys. Lett. B383 (1996) 48-53, http://arXiv.org/abs/hep-th/9512145.

[47] A. Giveon and D. Kutasov, "Little string theory in a double scaling limit," JHEP 10 (1999) 034, hep-th/9909110.

[48] F. Larsen and E. J. Martinec, "U(1) charges and moduli in the D1-D5 system," JHEP 06 (1999) 019, http://arXiv.org/abs/hep-th/9905064.

[49] J. A. Harvey, D. Kutasov, E. J. Martinec, and G. Moore, "Localized tachyons and RG flows," http://arXiv.org/abs/hep-th/0111154.

[50] E. J. Martinec, "Conformal field theory, geometry, and entropy," http://arXiv.org/abs/hep-th/9809021.

[51] L. J. Dixon, D. Friedan, E. J. Martinec, and S. H. Shenker, "The conformal field theory of orbifolds," Nucl. Phys. B282 (1987) 13-73.

[52] S. Hamidi and C. Vafa, "Interactions on orbifolds," Nucl. Phys. B279 (1987) 465.

[53] M. Bershadsky and A. Radul, "Conformal field theories with additional Z(n) symmetry," Int. J. Mod. Phys. A2 (1987) 165-178.

[54] O. Lunin and S. D. Mathur, "Correlation functions for M(n)/S(n) orbifolds," Commun. Math. Phys. 219 (2001) 399-442, http://arXiv.org/abs/hep-th/0006196.

[55] O. Lunin and S. D. Mathur, "Three-point functions for M(n)/S(n) orbifolds with N = 4 supersymmetry," Commun. Math. Phys. 227 (2002) 385-419, http://arXiv.org/abs/hep-th/0103169.

[56] A. Adams, J. Polchinski, and E. Silverstein, "Don't panic! closed string tachyons in ALE space-times," JHEP 10 (2001) 029, http://arXiv.org/abs/hep-th/0108075. 
[57] O. Coussaert, M. Henneaux, and P. van Driel, "The asymptotic dynamics of three-dimensional Einstein gravity with a negative cosmological constant," Class. Quant. Grav. 12 (1995) 2961-2966, http://arXiv.org/abs/gr-qc/9506019.

[58] J. Maldacena and H. Ooguri, "Strings in AdS(3) and SL(2,R) WZW model. I," hep-th/0001053.

[59] W. Fischler, S. Paban, and M. Rozali, "Collective coordinates in string theory," Phys. Lett. B352 (1995) 298-303, http://arXiv.org/abs/hep-th/9503072.

[60] A. Giveon and D. Kutasov, "Comments on double scaled little string theory," JHEP 01 (2000) 023, hep-th/9911039.

[61] D. Gershon, "Semiclassical versus exact solutions of charged black hole in four-dimensions and exact O(d,d) duality," Nucl. Phys. B421 (1994) 80-110, http://arXiv.org/abs/hep-th/9311122.

[62] J. Maldacena, G. Moore, and N. Seiberg, "Geometrical interpretation of D-branes in gauged WZW models," hep-th/0105038.

[63] S. Elitzur, A. Giveon, D. Kutasov, E. Rabinovici, and G. Sarkissian, "D-branes in the background of NS fivebranes," JHEP 08 (2000) 046, http://arXiv.org/abs/hep-th/0005052.

[64] V. Balasubramanian, S. F. Hassan, E. Keski-Vakkuri, and A. Naqvi, "A space-time orbifold: A toy model for a cosmological singularity," http://arXiv.org/abs/hep-th/0202187.

[65] B. Craps, D. Kutasov, and G. Rajesh, "String propagation in the presence of cosmological singularities," http://arXiv.org/abs/hep-th/0205101.

[66] G. Veneziano, "String cosmology: The pre-big bang scenario," http://arXiv.org/abs/hep-th/0002094.

[67] V. Balasubramanian, P. Kraus, A. E. Lawrence, and S. P. Trivedi, "Holographic probes of anti-de Sitter space-times," Phys. Rev. D59 (1999) 104021, http://arXiv.org/abs/hep-th/9808017.

[68] B. G. Carneiro da Cunha, "Inflation and holography in string theory," Phys. Rev. D65 (2002) 026001, http://arXiv.org/abs/hep-th/0105219.

[69] M. Spradlin and A. Strominger, "Vacuum states for $\operatorname{AdS}(2)$ black holes," JHEP 11 (1999) 021, http://arXiv.org/abs/hep-th/9904143.

[70] E. Keski-Vakkuri, "Bulk and boundary dynamics in BTZ black holes," Phys. Rev. D59 (1999) 104001, http://arXiv.org/abs/hep-th/9808037. 
[71] J. Louko, D. Marolf, and S. F. Ross, "On geodesic propagators and black hole holography," Phys. Rev. D62 (2000) 044041, http://arXiv.org/abs/hep-th/0002111.

[72] A. R. Steif, "The quantum stress tensor in the three-dimensional black hole," Phys. Rev. D49 (1994) 585-589, http://arXiv.org/abs/gr-qc/9308032.

[73] C. R. Nappi and E. Witten, "A closed, expanding universe in string theory," Phys. Lett. B293 (1992) 309-314, http://arXiv.org/abs/hep-th/9206078.

[74] S. W. Hawking, "The chronology protection conjecture," Phys. Rev. D46 (1992) 603-611. 